\title{
Relation of Obesity to Acute Myocardial Infarction in Japanese Patients
} Differences in Gender and Age

\author{
Jun Shiraishi, MD; Yoshio Kohno, MD; Takahisa Sawada, MD ${ }^{\dagger}$; Shinya Nishizawa, MD; \\ Masayasu Arihara, MD*; Mitsuyoshi Hadase, MD; Masayuki Hyogo, MD; \\ Takakazu Yagi, MD; Takatomo Shima, MD; Atsuko Nakazawa, MD**; \\ Masako Shigeta, MD**; Hiroyuki Yamada, $\mathrm{MD}^{\dagger}$; Tetsuya Tatsumi, $\mathrm{MD}^{\dagger}$; \\ Akihiro Azuma, $\mathrm{MD}^{\dagger}$; Hiroaki Matsubara, $\mathrm{MD}^{\dagger}$ \\ on Behalf of the AMI-Kyoto Multi-Center Risk Study Group
}

\begin{abstract}
Background It remains uncertain whether obesity is an independent risk factor for coronary heart disease in young adults, as well as adults, in Japan.

Methods and Results In the present study, 1,260 cases of acute myocardial infarction (AMI) and 3,775 community controls were recruited from the AMI-Kyoto Multi-Center Risk Study and Kyoto Citizen's Health and Nutrition Study, respectively. Obesity and other risk factors were retrospectively examined between cases and controls in each subgroup of young males (20-40 years), middle-aged males or females (40-60 years), older males or females (60-80 years), and very old males or females (80-100 years). In young, middle-aged, and older males, as well as in older females, cases had a higher body mass index (BMI) than controls. In young males, as well as in middle-aged and older females, cases had a higher prevalence of smoking than controls. Except for very old males, the prevalences of hypercholesterolemia, hypertension, and diabetes mellitus were higher in each subgroup of cases than in controls. Multivariate logistic regression analysis revealed that obesity (BMI $\geq 25)$ was an independent risk for AMI in young and middle-aged males, but not in females, whereas smoking was an independent risk for AMI in middle-aged and older females as well as in older males.

Conclusions Obesity is significantly associated with AMI, independent of the classic coronary risk factors, in young and middle-aged males. These findings support the current emphasis on controlling obesity to prevent coronary events in young Japanese male adults. (Circ J 2006; 70: 1525-1530)
\end{abstract}

Key Words: Acute myocardial infarction; Japan; Obesity; Risk factors; Smoking; Young adults

$\mathbf{O}$ besity is a risk factor for coronary heart disease (CHD) among adults ${ }^{1}$ and is increasing in prevalence among young adults, as well as adults, $2,3 \mathrm{~A}$ recent report indicated a significant association of obesity with coronary atherosclerosis in young male adults, particularly in those with a central pattern of adiposity, and little association of obesity with coronary atherosclerosis in young female adults. 4 Still another recent report points out that adiposity and its metabolic disturbances (ie, metabolic syndrome) are associated with early atherosclerotic change in adolescents? Recently we demonstrated that young Japanese patients with acute myocardial infarction (AMI) have a higher body mass index (BMI) compared with older patients, 6 but it remains uncertain whether obesity is a CHD

(Received May 19, 2006; revised manuscript received September 8, 2006; accepted September 29, 2006)

Departments of Cardiology, *Emergency Medicine, **Medical Checkup and Health Promotion, Kyoto First Red Cross Hospital and tDepartment of Cardiology and Vascular Regenerative Medicine, Kyoto Prefectural University School of Medicine, Kyoto, Japan

The participants in the AMI-Kyoto Multi-Center Risk Study Group are listed in Appendix 1.

Mailing address: Jun Shiraishi, MD, Department of Cardiology, Kyoto First Red Cross Hospital, Honmachi, Higashiyama-ku, Kyoto 605-0981, Japan. E-mail: sjun@msj.biglobe.ne.jp risk factor in young adults as well as older adults in Japan.

The AMI-Kyoto Multi-Center Risk Study, a large multicenter observational study in which 16 collaborating hospitals in Kyoto Prefecture have collected demographic, procedural, and outcome data on AMI patients, was established in 2000 in order to analyze these data and establish an emergency-hospital network for heart diseases in Kyoto6,7 The Kyoto Citizen's Health and Nutrition Study was performed among residents in Kyoto Prefecture, in order to examine behavior and lifestyle habits and develop effective public health intervention, 89 In the present study we used cases from the AMI-Kyoto Multi-Center Risk Study, and controls from the Kyoto Citizen's Health and Nutrition Study, respectively, to assess obesity as a risk factor for AMI in subgroups of 20-year age bands of each gender separately, based on a multi-center case-control study.

\section{Methods}

\section{Case and Control Subjects}

From January 2000 to June 2004, 1,651 consecutive patients with a diagnosis of AMI, who were admitted to AMIKyoto Multi-Center Risk Study Group Hospitals within 1 week after the onset of AMI, were enrolled in the present 


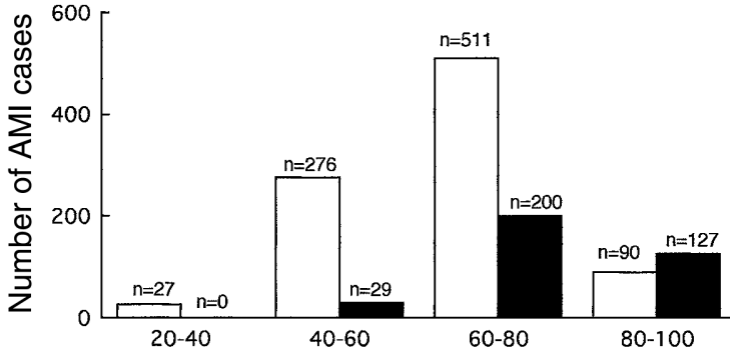

Fig 1. Number of acute myocardial infarction (AMI) cases by gender and age groups. Open bar, males; closed bar, females.

study. Of these, 1,260 AMI patients for whom the data concerning clinical characteristics and risk factors were available, were assigned as the case subjects. The diagnosis of AMI required the presence of 2 of the following 3 criteria: (1) characteristic clinical history, (2) serial changes on the ECG suggesting infarction (Q-waves) or injury (ST-segment elevations), and (3) transient increase in cardiac enzymes to more than 2-fold the normal laboratory values.

Control subjects comprised residents in Kyoto Prefecture who were involved in the Kyoto Citizen's Health and Nutrition Study as of November 1998.,9 We enrolled 3,775 consecutive individuals aged more than 20 years who did not have a history of cardiovascular, renal, or cerebrovascular diseases.

Both the case and control subjects were classified into 20-year age bands. We retrospectively examined obesity and other risk factors between cases and controls in each subgroup of young-aged males (20-40 years), middle-aged males or females (40-60 years), older males or females (60-80 years), and very old males or females (80-100 years).

\section{Data Collection}

The demographic information and risk factors for the cases were recorded. Obesity was defined as a BMI $\geq 25 \mathrm{~kg} / \mathrm{m}^{2}$. Hypercholesterolemia was defined as total cholesterol $\geq 220 \mathrm{mg} / \mathrm{dl}$ or the use of cholesterol-lowering agents; hypertension was defined as systemic blood pressure $\geq 140 / 90 \mathrm{mmHg}$ or a history of previous treatment; diabetes mellitus was defined as fasting blood sugar $\geq 126 \mathrm{mg} / \mathrm{dl}$ or the use of specific treatment. After informed consent to participate in the AMI-Kyoto Multi-Center Risk Study was given by each patient, all the in-hospital data were transmitted to the collection center located at the Department of Cardiology and Vascular Regenerative Medicine in Kyoto Prefectural University School of Medicine for analysis. The study protocol was approved by each hospital's ethics committee.

The personal characteristics, behavior, lifestyle habits, such as smoking status and alcohol consumption, and medical history of hypercholesterolemia, hypertension, diabetes mellitus, and other diseases of the controls were obtained from the questionnaires used in the Kyoto Citizen's Health and Nutrition Study 8,9 The questionnaires were mailed to residents at random and then collected by investigators of each health center in Kyoto Prefecture.

Data on BMI were available in all 27 young, 202 of 276 middle-aged, 367 of 511 older, and 58 of 90 very old males, and in 17 of 29 middle-aged, 139 of 200 older, and 83 of 127 very old females, and for all the control subjects.

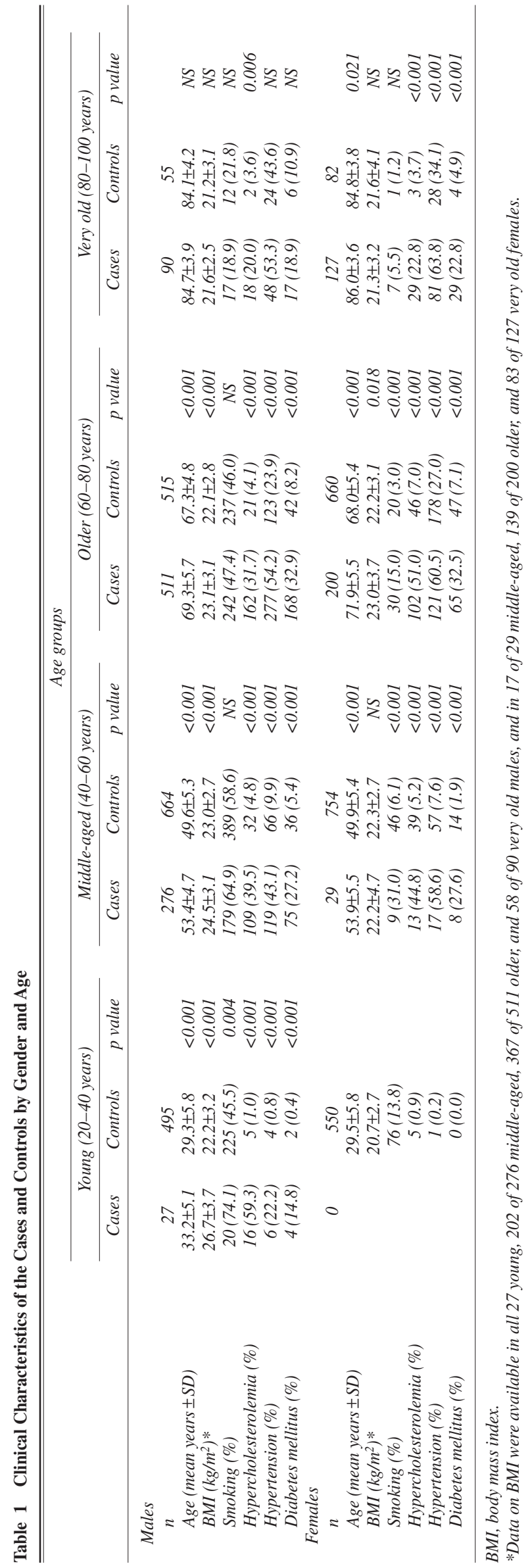

Circulation Journal Vol.70, December 2006 

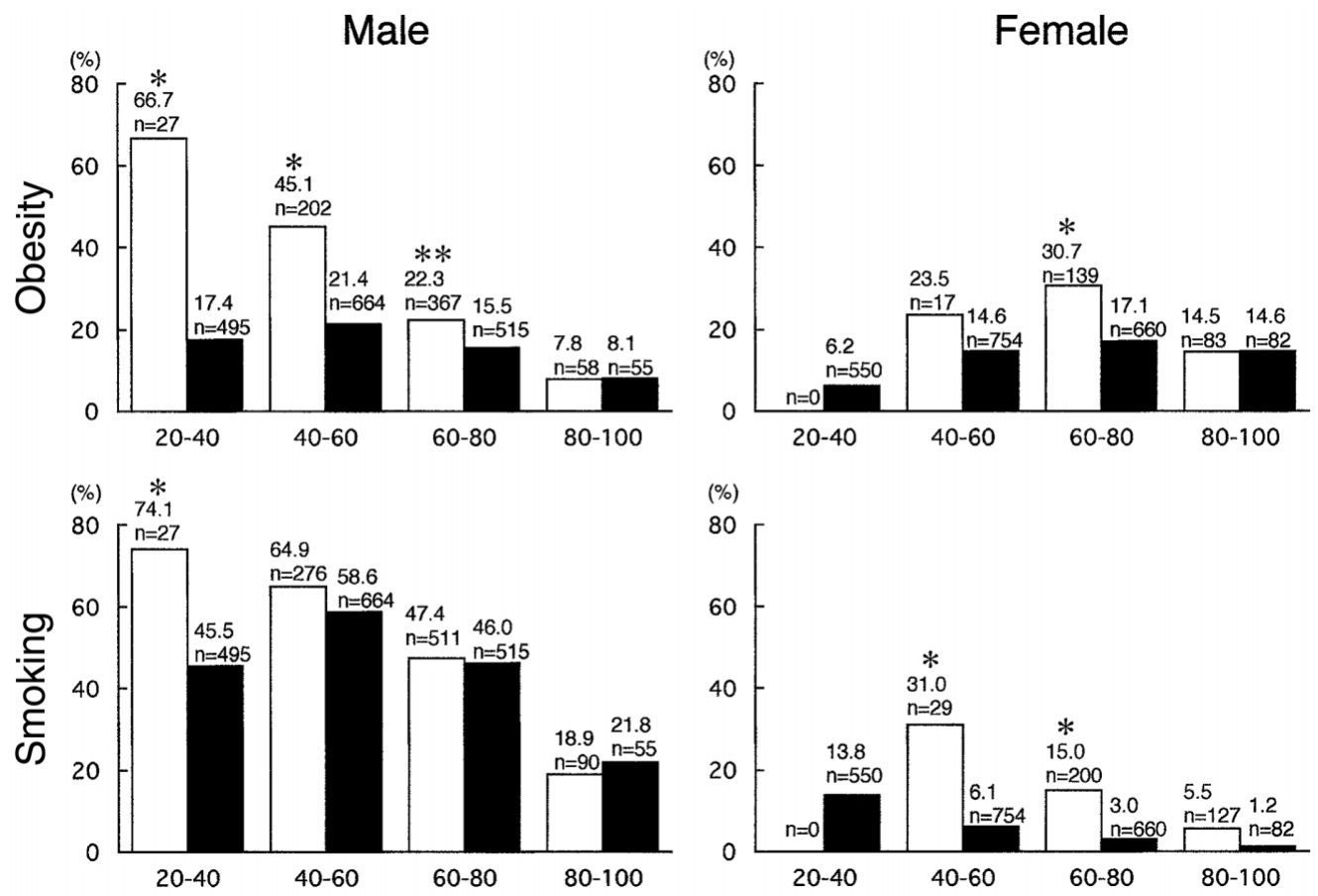

Fig 2. Prevalence of obesity and smoking by gender and age groups. Open bar, cases; closed bar, controls. *p<0.01 vs controls. $* * \mathrm{p}<0.05$ vs controls.
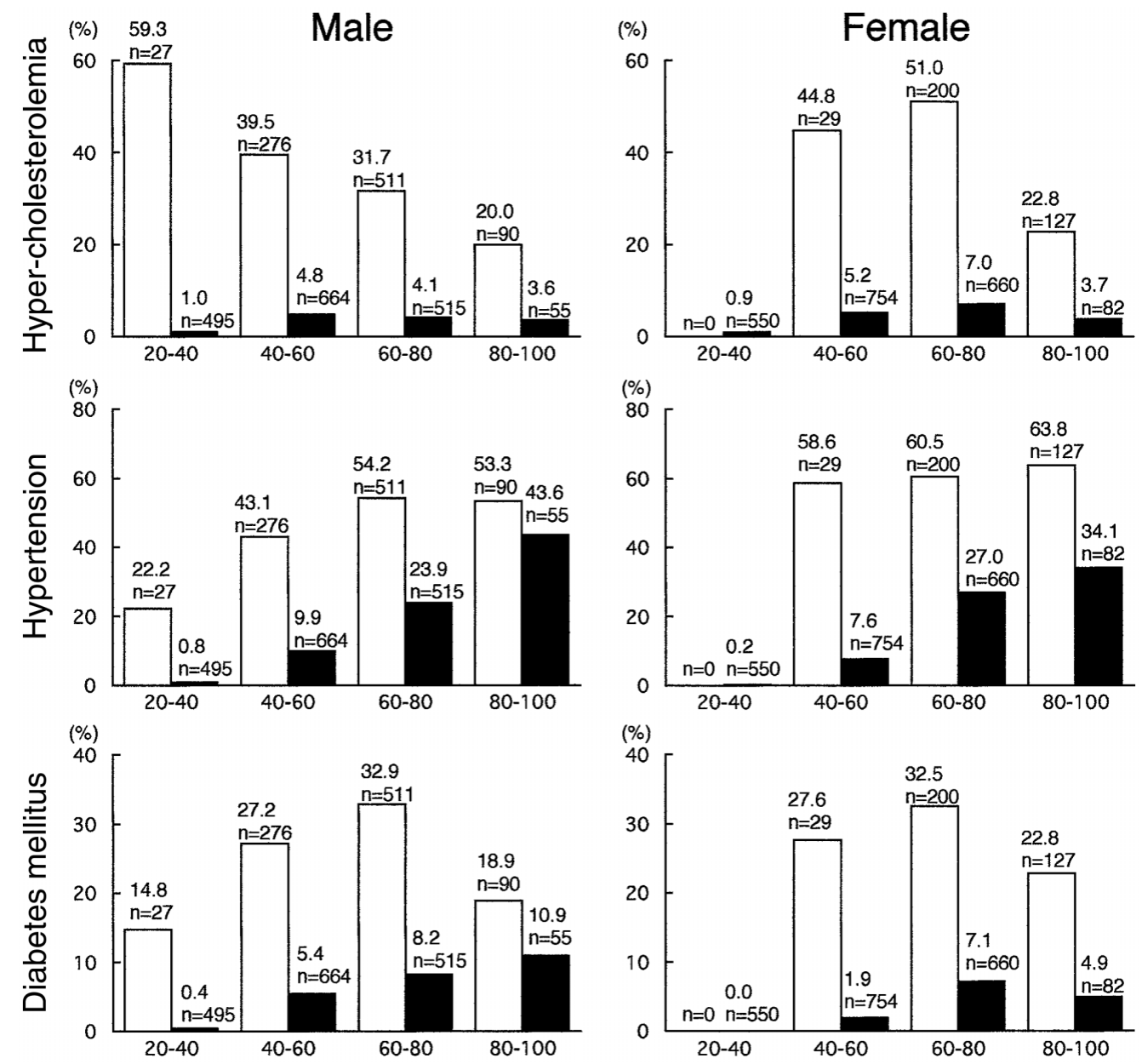

Fig 3. Prevalence of hypercholesterolemia, hypertension, and diabetes mellitus by gender and age groups. Open bar, cases; closed bar, controls. 
Table 2 Odds Ratio (OR) and 95\% Confidence Interval (CI) of Acute Myocardial Infarction According to Obesity or Smoking in Each Age Group

\begin{tabular}{|c|c|c|c|c|c|c|c|c|c|c|c|c|}
\hline \multirow{3}{*}{ Age group } & \multicolumn{6}{|c|}{ Obesity } & \multicolumn{6}{|c|}{ Smoking } \\
\hline & \multicolumn{3}{|c|}{ Male } & \multicolumn{3}{|c|}{ Female } & \multicolumn{3}{|c|}{ Male } & \multicolumn{3}{|c|}{ Female } \\
\hline & OR & $95 \% C I$ & $p$ value & $O R$ & $95 \% C I$ & p value & $O R$ & $95 \% C I$ & $p$ value & $O R$ & $95 \% C I$ & $p$ value \\
\hline 20-40 group & 4.730 & $1.391-16.081$ & 0.013 & & & & 0.990 & $0.303-3.231$ & 0.987 & & & \\
\hline $40-60$ group & 2.063 & $1.340-3.177$ & 0.001 & 1.143 & $0.289-4.515$ & 0.849 & 1.409 & $0.936-2.122$ & 0.101 & 16.679 & $4.338-64.132$ & $<0.001$ \\
\hline $60-80$ group & 1.228 & $0.794-1.898$ & 0.356 & 1.145 & $0.634-2.070$ & 0.653 & 1.576 & $1.132-2.194$ & 0.007 & 9.542 & $4.091-22.258$ & $<0.001$ \\
\hline 80-100 group & 1.395 & $0.371-5.252$ & 0.622 & 0.934 & $0.346-2.521$ & 0.892 & 0.918 & $0.340-2.477$ & 0.866 & 6.340 & $0.682-58.930$ & 0.104 \\
\hline
\end{tabular}

\section{Statistical Analysis}

Data are expressed as mean \pm SD. The cases and controls were compared using the chi-square test for discrete variables and unpaired Student's t-test for continuous variables according to standard statistical methods. The odds ratio and $95 \%$ confidence intervals assessing the risk of onset of AMI associated with obesity or smoking were estimated by multivariate logistic regression analysis of the case subjects having data on BMI and the control subjects. In all analyses, significance was accepted at $\mathrm{p}<0.05$.

\section{Results}

Fig 1 shows the number of AMI cases, as stratified by gender and age. There were no young females among them. The clinical characteristics and risk factors of the cases and the controls, as stratified by gender and age, are shown in Table 1, and Figs 2 and 3. Irrespective of gender, the case group was significantly older than the control group, except for very old males. The case group had a significantly greater BMI and a higher prevalence of obesity than the control group for young, middle-aged, and older males, as well as for older females. The case group had a significantly higher prevalence of smoking than the control group in young males, as well as in middle-aged and older females. Except for very old males, the prevalences of hypercholesterolemia, hypertension, and diabetes mellitus were significantly higher in the case group than in the control group of each gender and age group.

As shown in Table 2, obesity was a significant risk for AMI in young and middle-aged males, after adjustment for age, smoking, hypercholesterolemia, hypertension, and diabetes mellitus. In contrast, obesity was not associated with an increased risk for AMI in females or in older or very old males. Smoking was a significant risk for AMI in middleaged and older females, as well as in older males, after adjustment for age, obesity, hypercholesterolemia, hypertension, and diabetes mellitus (Table2). In contrast, smoking was not associated with an increased risk for AMI in young, middle-aged or very old males, or in very old females.

\section{Discussion}

The major findings of the present multicenter case-control study in Japan are: (1) obesity was an independent risk for AMI in young and middle-aged males, but not in females; and (2) smoking was an independent risk for AMI in middle-aged and older females, as well as in older males.

Although the data regarding the association of obesity with atherosclerosis and CHD have been inconsistent for many years ${ }^{10,11}$ recent accumulating evidence indicates that obesity is an independent CHD risk factor among adults! 1,12 There have also been conflicting data regarding the relationship between obesity and CHD in Japan! ${ }^{13-18}$ Some pro- spective studies have revealed that obesity is a CHD risk factor after adjusting for other risk factors, in Japan as well as in Western countries, ${ }^{13,14}$ but other case-control studies have not found the same result ${ }^{15-17}$ However, a recent casecontrol study has indicated that past obesity is a CHD risk factor, even after weight reduction! ${ }^{18}$ In the present report, we have also shown that obesity is a CHD risk factor in young and middle-aged males, but not in females or older males. The age- and gender-related differences in the CHD risk associated with obesity might contribute, at least in part, to these conflicting data.

As for young adults, there have also been inconsistent data regarding the effects of obesity on CHD. A previous prospective study in the United States showed that among males younger than 65, BMI had a significant positive association with the CHD risk, but the association was much weaker among older males aged 65 years or more ${ }^{19}$ In contrast, another case-control analysis of people aged 30-60 years in India indicated no association between BMI and $\mathrm{AMI}{ }^{20} \mathrm{~A}$ recent report indicated a significant relationship between obesity and coronary atherosclerosis in young male adults, particularly in those with central obesity, and little relationship between them in young female adults. Still other recent clinical studies have also shown that obesity and its metabolic impairment (ie, metabolic syndrome) are associated with impaired endothelial function and early atherosclerotic change in adolescents, 21,22 However, there are no data regarding the association of obesity with atherosclerosis and CHD in Japanese young adults. Recently, we showed that young Japanese patients with AMI have a higher BMI than older patients ${ }^{6}$ and in the present report, we have demonstrated for the first time that obesity is an independent AMI risk factor in young and middle-aged Japanese males.

In the present report, the susceptibility to AMI from the risk of obesity or smoking differed between males and females. A previous study has shown that the relative CHD risk associated with obesity in middle-aged and older females is slightly lower than that for males! $!^{2}$ The sex difference in the effect of obesity on CHD might be derived from the pattern of fat distribution. Although at an equivalent BMI, females at all ages have a larger percentage of body fat than male ${ }^{23}$ males are more prone to have a central (visceral) pattern of fat distribution ${ }^{24}$ Recent overwhelming evidence has indicated that adverse effects of obesity, such as a variety of metabolic disorders, cardiovascular morbidity, and mortality, are more tightly associated with central rather than peripheral obesity:5-27 Another possible explanation is the protective effects of female hormones against CHD progression in the premenopausal woman. In the present study, AMI cases had a significantly greater BMI than control subjects in the older female group, but there was no significant difference in BMI between AMI cases and control subjects among middle-aged females. We can 
not rule out the possibility that an increased prevalence of obesity after menopause, together with hypercholesterolemia, hypertension, and diabetes mellitus might also contribute, in part, to the etiology of CHD in older women.

On the other hand, our present study has indicated that smoking is a more independent and more significant risk factor for AMI in middle-aged and older females than in males, which is consistent with a recent report from Japanese investigators ${ }^{17}$ However, the mechanism for a gender difference in the effects of smoking remains to be elucidated. In general, females tend to be more susceptible to the respiratory disorders ascribed to smoking, such as asthma, bronchitis, and emphysema, than males ${ }^{28-30}$ Thus, it is reasonable to propose that females might be more sensitive to the effects of smoking on CHD than males.

Our results have shown that obesity is an independent risk for AMI in young and middle-aged males, not older or very old males. The reason for this age-related variability in the effect of obesity on AMI remains unclear. Older obese men may have a higher prevalence of hypertension or diabetes mellitus than younger obese ones. Thus, in the present study, adjusting for other risk factors might make the AMI risk associated with obesity more difficult to detect in the older men. Another possible explanation is that the increasing prevalence of obesity might make the risk easier to detect in young adults with $\mathrm{CHD}{ }^{31}$ The other possible explanation is that metabolic syndrome, accompanied by obesity, particularly visceral obesity, might play a central role in the pathogenesis of AMI in young male adults with a lower incidence of co-morbid diseases such as diabetes mellitus and hypertension. Findings from previous reports and our present study support the current emphasis on control of obesity, particularly visceral obesity, to prevent CHD in young adult males.

\section{Study Limitations}

First, there is a time-delay (approximately $1-4.5$ years) between the data for the case subjects and that for the control subjects. Second, there is a difference in the mean age (approximately 4 years or less) between the cases and the controls. Third, the present study population consisted of AMI patients who were admitted to collaborating hospitals and residents in Kyoto Prefecture, so it might not reflect the situation for the entire Japanese population. Fourth, data regarding risk factors and BMI were not available for all AMI patients. Further prospective studies or more precisely controlled case-control studies are needed to elucidate the CHD risk associated with obesity in Japan.

\section{Conclusion}

The present study provides evidence that obesity is an independent risk for AMI in young and middle-aged Japanese males, suggesting that it is necessary to correct obesity for primary prevention of AMI in young male adults in Japan. However, the relatively small sample size is a major limitation and a larger study should be performed to confirm our findings.

\section{Acknowledgment}

We thank Nobuo Shirahashi MD (Department of Industrial Medicine, Osaka City University Medical School) for advice and suggestions for the statistical analysis.

\section{References}

1. Jousilahti P, Tuomilehto J, Vartiainen E, Pekkanen J, Puska P. Body weight, cardiovascular risk factors, and coronary mortality: 15-year follow-up of middle-aged men and women in eastern Finland. Circulation 1996; 93: 1372-1379.

2. Kuczmarski RJ, Flegal KM, Campbell SM, Johnson CL. Increasing prevalence of overweight among US adults: The National Health and Nutrition Examination Surveys, 1960 to 1991. JAMA 1994; 272: $205-211$.

3. Troiano RP, Flegal KM, Kuczmarski RJ, Campbell SM, Johnson CL. Overweight prevalence and trends for children and adolescents: The National Health and Nutrition Examination Surveys, 1963 to 1991. Arch Pediatr Adolesc Med 1995; 149: 1085-1091.

4. McGill HC Jr, McMahan CA, Herderick EE, Zieske AW, Malcom GT, Tracy RE, et al. Obesity accelerates the progression of coronary atherosclerosis in young men. Circulation 2002; 105: 2712-2718.

5. Whincup PH, Gilg JA, Donald AE, Katterhorn M, Oliver C, Cook DG, et al. Arterial distensibility in adolescents: The influence of adiposity, the metabolic syndrome, and classic risk factors. Circulation 2005; 112: 1789-1797.

6. Shiraishi J, Kohno Y, Yamaguchi S, Arihara M, Hadase M, Hyogo $\mathrm{M}$, et al. Medium-term prognosis of young Japanese adults having acute myocardial infarction. Circ J 2006; 70: 518-524.

7. Shiraishi J, Kohno Y, Yamaguchi S, Arihara M, Hadase M, Hyogo $\mathrm{M}$, et al. Acute myocardial infarction in young Japanese adults: Clinical manifestations and in-hospital outcome. Circ J 2005; 69: $1454-$ 1458.

8. Tanaka K, Ikeda J, Higashi A, Nakazawa A, Nakatani M, Irie H, et al. The relationship between obesity and lifestyle in female residents: From Kyoto Citizen's Health and Nutrition Study 1998. Jpn J Nutr Diet 2002; 60: 195-202.

9. Tanaka K, Ikeda J, Higashi A, Nakazawa A, Nakatani M, Irie H, et al. Relationship between obesity and lifestyle of male citizens From Kyoto Citizen's Health and Nutrition Study 1998. Jpn J Nutr Diet 2003; 61: 195-204.

10. Keys A, Menotti A, Aravanis C, Blackburn H, Djordevic BS, Buzina R, et al. The seven countries study: 2,289 deaths in 15 years. Prev Med 1984; 13: 141-154.

11. Hubert HB, Feinleib M, McNamara PM, Castelli WP. Obesity as an independent risk factor for cardiovascular disease: A 26-year followup of participants in the Framingham Heart Study. Circulation 1983; 67: $968-977$.

12. Calle EE, Thun MJ, Petrelli JM, Rodriguez C, Heath CW Jr. Bodymass index and mortality in a prospective cohort of U.S. adults. $N$ Engl J Med 1999; 341: 1097-1105.

13. Kitamura A, Iso H, Naito Y, Iida M, Konishi M, Folsom AR, et al. High-density lipoprotein cholesterol and premature coronary heart disease in urban Japanese men. Circulation 1994; 89: 2533-2539.

14. Takahashi T, Chikamori T, Yonezawa Y, Sugimoto K, Yamada M, Takata J, et al. Prognostic value of serum cholesterol level in Japanese patients with coronary artery disease. Jpn Circ J 1997; 61: 139-144.

15. Miyake Y, Fukuoka Heart Study Group. Risk factors for non-fatal acute myocardial infarction in middle-aged and older Japanese. Jpn Circ J 2000; 64: 103-109.

16. Nakamura T, Tsubono Y, Kameda-Takemura K, Funahashi T, Yamashita S, Hisamichi S, et al. Magnitude of sustained multiple risk factors for ischemic heart disease in Japanese employees: A case-control study. Jpn Circ J 2001; 65: 11-17.

17. Kawano H, Soejima H, Kojima S, Kitagawa A, Ogawa H. Sex differences of risk factors for acute myocardial infarction in Japanese patients. Circ J 2006; 70: 513-517.

18. Washio M, Hayashi R. Past history of obesity (overweight by WHO criteria) is associated with an increased risk of nonfatal acute myocardial infarction: A case-control study in Japan. Circ J 2004; 68: $41-46$.

19. Rimm EB, Stampfer MJ, Giovannucci E, Ascherio A, Spiegelman D, Colditz GA, et al. Body size and fat distribution as predictors of coronary heart disease among middle-aged and older US men. Am J Epidemiol 1995; 141: 1117-1127.

20. Pais P, Pogue J, Gerstein H, Zachariah E, Savitha D, Jayprakash S, et al. Risk factors for acute myocardial infarction in Indians: A casecontrol study. Lancet 1996; 348: 358-363.

21. Watts K, Beye P, Siafarikas A, O'Driscoll G, Jones TW, Davis EA, et al. Effects of exercise training on vascular function in obese children. J Pediatr 2004; 144: 620-625.

22. Watts K, Beye P, Siafarikas A, Davis EA, Jones TW, O'Driscoll G, et al. Exercise training normalizes vascular dysfunction and improves central adiposity in obese adolescents. J Am Coll Cardiol 2004; 43: $1823-1827$. 
23. Gallagher D, Visser M, Sepulveda D, Pierson RN, Harris T, Heymsfield SB. How useful is body mass index for comparison of body fatness across age, sex, and ethnic groups? Am J Epidemiol 1996; 143: 228-239.

24. Lemieux S, Prud'homme D, Bouchard C, Tremblay A, Despres JP. Sex differences in the relation of visceral adipose tissue accumulation to total body fatness. Am J Clin Nutr 1993; 58: 463-467.

25. Folsom AR, Kaye SA, Sellers TA, Hong CP, Cerhan JR, Potter JD, et al. Body fat distribution and 5-year risk of death in older women. JAMA 1993; 269: $483-487$.

26. Lemieux S, Despres JP, Moorjani S, Nadeau A, Theriault G, Prud'homme D, et al. Are gender differences in cardiovascular disease risk factors explained by the level of visceral adipose tissue? Diabetologia 1994; 37: 757-764.

27. Duncan BB, Chambless LE, Schmidt MI, Szklo M, Folsom AR, Carpenter MA, et al. Correlates of body fat distribution: Variation across categories of race, sex, and body mass in the atherosclerosis risk in communities study. Ann Epidemiol 1995; 5: 192-200.

28. Baldini EH, Strauss GM. Women and lung cancer: Waiting to exhale. Chest 1997; 112: 229-234.

29. Chen Y, Dales R, Krewski D, Breithaupt K. Increased effects of smoking and obesity on asthma among female Canadians: The National Population Health Survey, 1994-1995. Am J Epidemiol 1999; 150: $255-262$.

30. Chen Y, Breithaupt K, Muhajarine N. Occurrence of chronic ob- structive pulmonary disease among Canadians and sex-related risk factors. J Clin Epidemiol 2000; 53: 755-761.

31. Imamura H, Izawa A, Kai R, Yokoseki O, Uchikawa S, Yazaki Y, et al. Trends over the last 20 years in the clinical background of young Japanese patients with coronary artery disease. Circ J 2004; 68: $186-191$.

\section{Appendix 1}

The following institutions and principal investigators participated in the present study as the AMI-Kyoto Multi-Center Risk Study Group.

Kyoto Municipal Hospital: Furukawa K, Matsubara K, Ashida T; Kyoto Kidugawa Hospital: Miyanaga H, Nakagawa T, Matsui H; Kyoto Second Red Cross Hospital: Kitamura M, Inoue N, Fujita H, Tanaka T, Inoue K, Matsuo A, Suzuki K; Social Insurance Kyoto Hospital: Yamada C, Oda Y, Inoue M; Rakuwakai Marutamachi Hospital: Kusuoka S, Katamura M; Nantan General Hospital: Okada T; Kameoka Municipal Hospital: Kuriyama T; Ayabe Municipal Hospital: Shiga K, Kohno Y; Maizuru Medical Center: Harada Y, Yamanaka S; Kyoto Saiseikai Hospital: Yamahara Y, Ishibashi K, Kuroyanagi A; Gakkentoshi Hospital: Sakai R, Akashi K; Kouseikai Takeda Hospital: Ito K, Kinoshita N; Kyoto Prefectural Yosanoumi Hospital: Keira N, Kimura S; Fukuchiyama Municipal Hospital: Yamamoto K; Kyoto Prefectural University School of Medicine: Shirayama T, Matoba S, Tsutsumi Y, Takahashi T, Shiraishi H, Nakamura $\mathrm{T}$. 